\title{
Anxiety and Related Factors in Parents About Coronavirus Disease-2019 for Children
}

\author{
๑ Şeyda Binay Yaz1', ๑ Ayşe Kahraman², ๑ Merve Gümüş̧2 \\ 1ं izmir Bakırçay University Faculty of Health Sciences, Department of Nursing, Division of Pediatric Nursing, İzmir, Turkey \\ Ege University Faculty of Nursing, Department of Pediatric Nursing, İzmir, Turkey
}

\begin{abstract}
Aim: The aim of study was to determine the anxiety and related factors in parents about new Coronavirus disease-2019 (COVID-19).

Materials and Methods: The sample of the descriptive study consisted of 494 parents with children aged 0-18. The data were collected during April-May 2020 with tools that were prepared via Google Docs, an online study. The link to the questionnaires was shared via social media. The volunteers were agreed to participate in the survey.

Results: In the study, $94.5 \%$ of the parents participating are mothers, $69.5 \%$ are between the ages of $31-40$ and $59.6 \%$ have one child. $31.8 \%$ of the parents stated that they worked during the COVID-19 pandemic, $90.3 \%$ applied social isolation or quarantine. The most frequent feeling that $83.6 \%$ of the parents was concern/anxiety, $69.0 \%$ used kitchen activities such as cooking and baking cakes as coping mechanisms, $68.4 \%$ used games, and painting activities with children.

Conclusion: In this study, it was determined that parents' anxiety levels were mild. It is recommended that social and health initiatives be created to prevent and alleviate the psychosocial effects of the pandemic, and to develop programs that will reduce parents' anxiety.
\end{abstract}

Keywords: COVID-19, pandemic, anxiety, parent, children

\section{Introduction}

Coronavirus disease-2019 (COVID-19) was identified as a disease which caused respiratory problems in December 2019 (1). This virus has a high and rapid contagiousness. It is transmitted from person to person (2). The World Health Organization (WHO) declared a coronavirus to be a pandemic on March 11 1 th 2020, and named this disease 2019-new coronavirus (2019-nCoV) $(3,4)$. In Turkey, COVID-19 started to be seen as of March 19, 2020. The WHO recommended that millions of people "stay home and socially isolated to avoid" COVID-19 transmission (5). The Ministry of Health in Turkey has also launched a stay-at-home campaign with the motto "Life Fits into Home" immediately after the incidents began to appear in our country. In this context, the whole community started to stay at home except for mandatory needs (6). The effects of infectious diseases-related outbreaks and social isolation on community mental health were included in many studies (7-10). It was stated that such outbreaks could cause psychological situations. These can include stress, depression, fear, and anxiety $(10,11)$.

In China, the first country affected by coronavirus, people were reported to show signs of stress, depression, and anxiety (12). Especially in those people who were in quarantine, fear, irritability, sadness, and feelings of guilt are found to be 
common negative emotions during this process (13). No study investigating the effects of the COVID-19 pandemic on the child and family were found in the literature. In a simple study, it was stated that families face stress factors such as commuting to work, bringing home viruses, and meeting their children's increased educational and care needs (14). Mothers and fathers should pay attention to their children's health and illnesses. Besides this, they have to deal with some of the uncertainties surrounding their family during COVID-19 isolation (15). Nurses, as well as families, have important roles and responsibilities in the protection and development of children, family, and community health (16). Also, nurses working in the field of paediatric nursing, family health nursing or public health nursing should have a responsibility for the management of the health of the family.

It is thought that the global COVID-19 outbreak will lead to deep psychological effects on families. Evidence-based strategies should be developed to reduce any negative psychological effects, psychiatric symptoms, or anxiety during the epidemic. Determining the anxiety levels and their related factors experienced by children who are one of the risk groups in infectious diseases, and therefore their families is important for better management of the situation. This study was carried out to identify the anxiety and related factors of parents associated with COVID-19.

\section{Materials and Methods}

This study has a descriptive design. The data was collected with an online survey during April and May 2020. The increase in the number of people using the Internet means that researchers have the opportunity to recruit participants from different backgrounds and different geographical regions that would not otherwise be possible (17). As it helps to reach the hard-to-reach segments of the general population by taking advantage of this feature of the Internet, also with the effect of the pandemic, the link for the questionnaires was shared via social networking sites such as Facebook, Twitter, Instagram, blogs, and forums.

The focus of the research was parents who have children between 0-18 years old, and also use social media and agreed to participate in the research. Since the number of elements in the universe is unknown, the number of samples was calculated with the formula $n=t 2 p q / d 2$ ( $n=$ number of individuals to be sampled, $t=1.96, p=0.50$, $q=050, d=0.05)$. According to this formula, the sample size was determined to be at least 385 (95\% confidence interval in the calculation, the $\alpha$ value for the significance level is taken to be 0.05) (18). The number of parents included in the study was 498 . Four parents who were not voluntary or who answered the questionnaire incomplete were excluded from the sample. The final sample was 494 parents.

\section{Ethical Considerations}

İzmir Bakırçay University, Non-Interventional Clinical Research Ethics Committee's written permission was taken (approval number: 21/21, approval date: 20.04.2020) to conduct the research.

The consents of the individuals were taken and then they were allowed to answer the questions after the consent.

\section{Data Collection}

The data collection tools were prepared using the Google Docs website. The study was conducted via the internet by means of social media. The social media tools were Facebook, Twitter, Instagram, blogs, and forums. The link for the questionnaires was distributed. The parents agreed to participate in the survey voluntarily.

Information Form Introducing the Child and Family: This is a form consisting of 8 questions including questions regarding the sociodemographic characteristics (age, gender, education, etc.) of the child and the family.

Questionnaire of Anxiety and Related Factors: There are 14 questions in this form that include anxiety factors related to coronavirus using the literature. The questions are about the symptoms of infectious diseases of children and other family members, social isolation, frequent feelings and coping skills of the parents, transportation preferences, and prevention methods (19-21).

Beck Anxiety Inventory: This twenty-one item Likerttype self-assessing scale was developed for anxiety by Beck in 1988. Likert (sum of degrees) provides type measurement. There are 4 options in each of the twenty-one symptom categories. Each item is given points between 0 and 3. The person is asked to assess the signs within the last week including the day of the questionnaire. Each symptom is evaluated as none, mild, moderate, or severe. The final score ranges from 0 to 63 . The value of the score obtained from the scale shows the anxiety experienced by an individual. The Turkish language validity and reliability study of this scale were conducted. A result of 8-15 points shows mild anxiety, 16-25 shows moderate anxiety, and 26-63 shows severe anxiety. The Cronbach's alpha value for this scale was 0.93 . The scale has two subgroups: "Subjective Anxiety" and "Somatic Symptoms". The subjective anxiety sub-dimension contains 13 items $(1,4,5,7-11,14-17,19)$ and the score is between 
0 and 39, the somatic symptoms sub-dimension is 8 items $(2,3,6,12,13,18,20,21)$ and its score varies between 0 and 24 (22).

\section{Statistical Analysis}

Analysis of the data to be obtained from the research was carried out through the SPSS 26.0 package program. Descriptive statistics in the analysis of the research data were calculated as a number, percentage, average, and standard deviation. Additionally, Shapiro-Wilk/Kolmogorov-Smirnov (K-S) was carried out to determine the suitability of the data for normal distribution. If the data were suitable for normal distribution, Student's t-test, and One-Way ANOVA were used. If it was not suitable for normal distribution, the Mann-Whitney U test, and the Kruskal-Wallis test were used. Multiple linear regressions analysis was performed to determine the effect of various independent variables on the parents' anxiety level scores during the pandemic process. The results are presented as estimated $\beta$ (Standard Error), p-values, and R2 value. The significance level was accepted as 0.05 .

\section{Validity, Reliability, and Rigour}

The data instruments were selected by the researchers for their relevance to the study population. All data collection tools demonstrated reliability and validity for the Turkish population. The researchers received preliminary online education on Google Documents data collection. Interpretation of the survey content was also included in the education content to ensure the accuracy and consistency of the data.

\section{Results}

Almost all the 494 participants were mothers (94.5\%) and the average age of $69.5 \%$ was between $31-40$ years. Sixtypoint nine percent of the participants were bachelor, $67.8 \%$ were working, $45.3 \%$ of the income assessment was in the medium level. $56.9 \%$ had 1 child and $37.3 \%$ had 2 children. The Beck Anxiety Inventory mean score of the participants was found to be $11.77 \pm 10.36$ and this was considered as mild level. The Subjective Anxiety Subscale mean score was $8.93 \pm 7.47$, and the Somatic symptoms subscale mean score was $2.84 \pm 3.42$ (Table I).

Before the COVID-19 pandemic, 95.1\% had never been to another country and $92.5 \%$ had never had any guests from another country. During the COVID-19 pandemic, $83.4 \%$ of the parents took care of their children themselves. $33.6 \%$ of participants had a family member with a chronic disease. A statistically significant difference was found between family members with and without chronic disease $(p=0.003)$. The anxiety score average of those with chronic diseases in their family was found to be higher. $87.0 \%$ of participants did not have a condition requiring constant hospitalization (follow-up, treatment, etc.), only $8.5 \%$ had a person who was constantly taking medication within their family members. A statistically significant difference was found between those families with or without members taking regular medication $(p=0.047)$. The anxiety score average for those with a member of their family taking medication was found to be higher. $75.3 \%$ of parents had not received training on infectious diseases and prevention methods. During the COVID-19 pandemic, $90.3 \%$ of participants implemented social isolation or quarantine (Table II).

Considering the participants' use of vehicles during the pandemic, 83.6\% used their own private car for transportation. A statistically significant difference was found between those who used private cars and those who did not $(p=0.042)$. The anxiety score average of those who used their private car was found to be lower. According to information source used to obtain developments regarding COVID-19, it was reported that $90.7 \%$ of participants preferred the internet/social media, while $56.5 \%$ preferred television/radio. While there was no significant difference between those who preferred the internet-social media as a news source about COVID-19 and those who did not $(p=0.105)$, a difference was found between those who preferred TV-radio and those who did not $(p<0.001)$. The anxiety score average of those who preferred TV-radio was found to be lower. In the first place was the difference between those participants who preferred using a mask (96.0\%) and keeping a social-distance (1 meter) as a protection method. The most common emotions about COVID-19 experienced by parents were concern/anxiety $(83.6 \%)$, fear (38.5\%), sadness (33.8\%), and difficulty in coping $(21.7 \%)$. When the parents' anxiety scores were compared with emotions such as fear, sadness, and difficulty in coping, a statistically significant difference was found between those who experienced these feelings and those who did not $(p<0.001, p<0.001, p<0.001)$. The anxiety score average of those parents who experienced these emotions was higher than those who did not. The parents' methods of coping during social isolation included kitchen activities (cooking, baking cake, etc.) (69.0\%), games and painting with their children (68.4\%), home cleaning $(65.8 \%)$, and reading $(40.5 \%)$. A statistically significant difference was found between those who preferred the reading method and those who did not $(p=0.034)$. The 
anxiety score average of those who preferred to read books was lower (Table III).

Several factors could be seen to significantly affect the anxiety scores of the parents in the pandemic when regression analysis was performed; the employment status of the parents, the presence of an individual with a chronic disease in the family, the person caring for the child during the pandemic process, the most common feelings of anxiety, fear, sadness, and difficulties in coping are included in these. These variables explain $23 \%$ of the anxiety level of the parents during the COVID-19 pandemic (Table IV).

Table I. Comparison of participants' socio-demographic characteristics and anxiety

\begin{tabular}{|c|c|c|c|c|c|c|}
\hline \multirow[t]{2}{*}{ Variables } & \multirow{2}{*}{$\begin{array}{l}N \\
494 \\
\end{array}$} & \multirow{2}{*}{\begin{tabular}{|l|}
$\%$ \\
100 \\
\end{tabular}} & \multirow{2}{*}{$\begin{array}{l}\mathrm{BAI} \\
\mathrm{X} \pm \mathrm{SD}\end{array}$} & \multicolumn{3}{|l|}{ Significance } \\
\hline & & & & Mean Rank & Test & p-value \\
\hline \multicolumn{6}{|l|}{ Parents } & \multirow{3}{*}{$<0.001$} \\
\hline Mother & 467 & 94.5 & $12.15 \pm 0.48$ & 253.41 & \multirow{2}{*}{$Z=-3,834$} & \\
\hline Father & 27 & 5.5 & $5.29 \pm 1.20$ & 145.20 & & \\
\hline \multicolumn{6}{|l|}{ Age } & \multirow{4}{*}{0.220} \\
\hline $19-30$ & 58 & 11.7 & $13.01 \pm 1.43$ & 264.12 & \multirow{3}{*}{$\chi^{2}=3,026$} & \\
\hline $31-40$ & 343 & 69.5 & $11.85 \pm 0.55$ & 250.48 & & \\
\hline 41 and above & 93 & 18.8 & $10.70 \pm 1.10$ & 226.13 & & \\
\hline \multicolumn{6}{|l|}{ Education } & \multirow{5}{*}{0.885} \\
\hline First-secondary education & 5 & 1.0 & $11.40 \pm 3.58$ & 258.00 & \multirow{4}{*}{$x^{2}=0.650$} & \\
\hline High school & 46 & 9.3 & $11.73 \pm 1.71$ & 235.55 & & \\
\hline Bachelor & 301 & 60.9 & $12.03 \pm 0.60$ & 251.05 & & \\
\hline Graduate & 142 & 28.8 & $11.25 \pm 0.80$ & 243.47 & & \\
\hline \multicolumn{6}{|l|}{ Profession } & \multirow{4}{*}{0.954} \\
\hline Unemployed & 55 & 11.1 & $11.41 \pm 1.25$ & 247.79 & \multirow{3}{*}{$\chi^{2}=0.094$} & \\
\hline Health employee & 20 & 4.0 & $10.75 \pm 2.10$ & 237.95 & & \\
\hline Other professional groups & 419 & 84.9 & $11.87 \pm 0.51$ & 247.92 & & \\
\hline \multicolumn{6}{|l|}{ Working status } & \multirow{3}{*}{0.002} \\
\hline Working & 335 & 67.8 & $10.73 \pm 9.83$ & 233.49 & & \\
\hline Not working & 159 & 32.2 & $13.96 \pm 11.11$ & 277.02 & $Z=-3,1 / 0$ & \\
\hline \multicolumn{6}{|l|}{ Economic situation assessment } & \multirow{4}{*}{0.070} \\
\hline Low level & 104 & 21.1 & $14.52 \pm 1.21$ & 276.01 & \multirow{3}{*}{$x^{2}=5,326$} & \\
\hline Intermediate level & 224 & 45.3 & $11.25 \pm 0.66$ & 241.39 & & \\
\hline High level & 166 & 33.6 & $10.75 \pm 0.70$ & 237.88 & & \\
\hline \multicolumn{6}{|l|}{ Number of children } & \multirow{4}{*}{0.173} \\
\hline 1 & 281 & 56.9 & $12.11 \pm 0.61$ & 255.58 & \multirow{3}{*}{$\chi^{2}=3,512$} & \\
\hline 2 & 187 & 37.8 & $11.03 \pm 0.77$ & 232.50 & & \\
\hline 3 and above & 26 & 5.3 & $12.76 \pm 1.84$ & 268.04 & & \\
\hline Beck Anxiety Inventory mean score & 494 & 100.0 & $11.77 \pm 10.36$ & \multicolumn{3}{|c|}{$\begin{array}{l}9.00(0-53) \\
\text { Median (Min.-Max.) }\end{array}$} \\
\hline Subjective Anxiety Subscale mean score & 494 & 100.0 & $8.93 \pm 7.47$ & \multicolumn{3}{|c|}{$\begin{array}{l}7.00(0-34) \\
\text { Median (Min.-Max.) }\end{array}$} \\
\hline Somatic Symptoms Subscale mean score & 494 & 100.0 & $2.84 \pm 3.42$ & \multicolumn{3}{|c|}{$\begin{array}{l}2.00(0-19) \\
\text { Median (Min.-Max.) }\end{array}$} \\
\hline
\end{tabular}




\section{Discussion}

It has been seen that there has been a rapid rise in the number of cases and deaths in the COVID-19 pandemic in the world. This situation has led to psychological effects such as stress, depression, and anxiety in people. Fear of uncertainty has been reported to cause negative behaviour (23). It was also emphasized with the proposal of $\mathrm{WHO}$ that the quarantine decisions and curfews made by most countries might increase stress, anxiety, and depression $(13,24)$. It was found that the quarantine measures applied in the severe acute respiratory syndrome and Middle East respiratory syndrome (MERS) outbreaks were associated with psychosocial problems. These were depressive signs, post-traumatic stress, anxiety, stress, social isolation, loneliness, and stigma, and the most important determinants were the length of the quarantine and loss of income (25).
Also, insufficient and fake news about COVID-19 has led to increased anxiety and fear regarding the situation in this process (2). The WHO suggested that people should only receive news on COVID-19 from reliable sources. In this study, most of the participants used social media as their news source. However, the anxiety scores of those who preferred TV news as their news source were found to be lower.

In a study conducted on 1,210 people in China examining the psychological effects of coronavirus, it was reported that $28.8 \%$ of the people in the community showed moderate or severe anxiety, $16.5 \%$ showed moderate or severe depression, and $8.1 \%$ showed moderate or severe stress (12). A study in Canada found that one-third of 1,354 Canadian adults were concerned about the coronavirus and 7\% were "very worried" (26). In Spain, while the signs of depression, stress, and anxiety were low in the early stages of the

\begin{tabular}{|l|l|l|l|l|l|l|l|l|l|}
\hline Table II. Comparison of participants' COVID-19 characteristics and anxiety scores & $\mathbf{N}$ & $\mathbf{\%}$ & BAI & \multicolumn{3}{|l|}{ Significance } \\
\hline Variables & $\mathbf{4 9 4}$ & $\mathbf{1 0 0}$ & $\mathbf{X} \pm$ SD & Mean Rank & Test & p-value \\
\hline \multicolumn{7}{|l|}{} \\
\hline Going to another country before the COVID-19 pandemic & $\begin{array}{l}24 \\
470\end{array}$ & $\begin{array}{l}4.9 \\
95.1\end{array}$ & $\begin{array}{l}8.79 \pm 2.07 \\
11.92 \pm 047\end{array}$ & $\begin{array}{l}194.15 \\
250.22\end{array}$ & Z=-1,879 & 0.060 \\
\hline $\begin{array}{l}\text { Yes } \\
\text { No }\end{array}$ &
\end{tabular}

Guest arrival from another country before COVID-19 pandemic

\begin{tabular}{|c|c|c|c|c|c|c|}
\hline $\begin{array}{l}\text { Yes } \\
\text { No }\end{array}$ & $\begin{array}{l}37 \\
457\end{array}$ & $\begin{array}{l}7.5 \\
92.5\end{array}$ & $\begin{array}{l}12.21 \pm 1.41 \\
11.74 \pm 0.49\end{array}$ & $\begin{array}{l}266.09 \\
245.99\end{array}$ & $Z=-0.825$ & 0.410 \\
\hline \multicolumn{7}{|c|}{ Caregiver for children in the COVID-19 pandemic } \\
\hline $\begin{array}{l}\text { Mother/Father } \\
\text { Caregiver } \\
\text { Grandma/grandfather } \\
\text { Other (family elders, relatives, etc.) }\end{array}$ & $\begin{array}{l}412 \\
28 \\
46 \\
8\end{array}$ & $\begin{array}{l}83.4 \\
5.7 \\
9.3 \\
1.6\end{array}$ & $\begin{array}{l}11.98 \pm 0.51 \\
9.21 \pm 1.49 \\
11.00 \pm 1.67 \\
14.50 \pm 3.84\end{array}$ & $\begin{array}{l}250.90 \\
220.57 \\
226.08 \\
290.00\end{array}$ & $\chi^{2}=2,982$ & 0.394 \\
\hline
\end{tabular}

Presence of chronic disease in family members (Diabetes, heart disease, kidney disease, etc.)

\begin{tabular}{|l|l|l|l|l|l|l|}
\hline Yes & 166 & 33.6 & $13.73 \pm 0.86$ & 273.92 & & $Z=-2,930$ \\
No & 328 & 66.4 & $10.78 \pm 0.54$ & 234.13 & 0.003 \\
\hline
\end{tabular}

Presence of a condition requiring constant hospitalization in family members (follow-up, treatment, etc.)

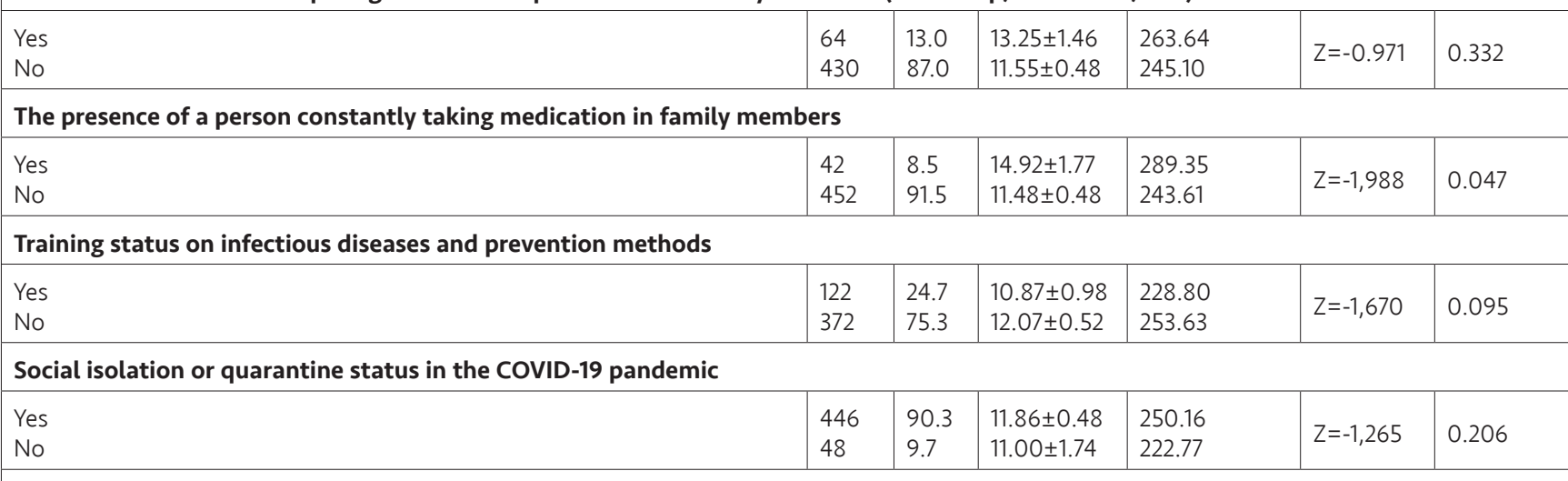

X: Average, SD: Standard deviation, BAI: Beck anxiety inventory, COVID-19: Coronavirus disease-2019 
Table III. Comparison of participants' coping methods and problems during the pandemic and their anxiety scores

\begin{tabular}{|c|c|c|c|c|c|c|c|}
\hline \multirow[t]{2}{*}{ Variables } & & \multirow{2}{*}{$\begin{array}{l}N \\
494\end{array}$} & \multirow{2}{*}{$\begin{array}{l}\% \\
100 \\
\end{array}$} & \multirow{2}{*}{$\begin{array}{l}\text { BAI } \\
X \pm S D\end{array}$} & \multicolumn{3}{|l|}{ Significance } \\
\hline & & & & & Mean Rank & Test & p-value \\
\hline \multicolumn{8}{|l|}{ Type of transportation use } \\
\hline Personal car & $\begin{array}{l}\text { Yes } \\
\text { No }\end{array}$ & $\begin{array}{l}413 \\
81 \\
\end{array}$ & \begin{tabular}{|l|}
83.6 \\
16.4 \\
\end{tabular} & $\begin{array}{l}11.27 \pm 0.49 \\
11.93 \pm 1.28\end{array}$ & $\begin{array}{l}241.73 \\
276.93 \\
\end{array}$ & $Z=-2,032$ & 0.042 \\
\hline Public transportation (bus, minibus) & $\begin{array}{l}\text { Yes } \\
\text { No }\end{array}$ & $\begin{array}{l}24 \\
470\end{array}$ & $\begin{array}{l}4.9 \\
95.1 \\
\end{array}$ & $\begin{array}{l}10.91 \pm 2.50 \\
11.82 \pm 0.47\end{array}$ & $\begin{array}{l}221.38 \\
248.83\end{array}$ & $Z=-0.920$ & 0.357 \\
\hline Taxi & $\begin{array}{l}\text { Yes } \\
\text { No }\end{array}$ & $\begin{array}{l}14 \\
480 \\
\end{array}$ & \begin{tabular}{|l|}
2.8 \\
97.2 \\
\end{tabular} & $\begin{array}{l}13.57 \pm 3.18 \\
11.72 \pm 0.47\end{array}$ & $\begin{array}{l}267.46 \\
246.92 \\
\end{array}$ & $Z=-0.531$ & 0.595 \\
\hline Other (bicycle, motorcycle, subway) & $\begin{array}{l}\text { Yes } \\
\text { No }\end{array}$ & $\begin{array}{l}12 \\
482\end{array}$ & $\begin{array}{l}2.4 \\
97.6 \\
\end{array}$ & $\begin{array}{l}12.08 \pm 3.37 \\
11.76 \pm 0.47\end{array}$ & $\begin{array}{l}239.75 \\
247.69 \\
\end{array}$ & $Z=-0.191$ & 0.849 \\
\hline \multicolumn{8}{|c|}{ The information source of developments regarding COVID-19 } \\
\hline Internet-social media & $\begin{array}{l}\text { Yes } \\
\text { No }\end{array}$ & $\begin{array}{l}448 \\
46 \\
\end{array}$ & \begin{tabular}{|l|}
90.7 \\
9.3 \\
\end{tabular} & $\begin{array}{l}11.87 \pm 0.47 \\
10.80 \pm 1.81\end{array}$ & \begin{tabular}{|l|}
250.83 \\
215.09 \\
\end{tabular} & $Z=-1,619$ & 0.105 \\
\hline Television-radio & $\begin{array}{l}\text { Yes } \\
\text { No }\end{array}$ & $\begin{array}{l}279 \\
215\end{array}$ & $\begin{array}{l}56.5 \\
43.5 \\
\end{array}$ & $\begin{array}{l}10.48 \pm 0.59 \\
13.46 \pm 0.72\end{array}$ & $\begin{array}{l}227.27 \\
273.75\end{array}$ & $Z=-3,592$ & $<0.001$ \\
\hline Health professionals & $\begin{array}{l}\text { Yes } \\
\text { No }\end{array}$ & $\begin{array}{l}122 \\
372 \\
\end{array}$ & $\begin{array}{l}24.7 \\
75.3 \\
\end{array}$ & $\begin{array}{l}11.22 \pm 1.00 \\
11.95 \pm 0.52\end{array}$ & \begin{tabular}{|l|}
234.47 \\
251.77 \\
\end{tabular} & $Z=-1,163$ & 0.245 \\
\hline Other (newspaper, friend, neighbor) & $\begin{array}{l}\text { Yes } \\
\text { No }\end{array}$ & $\begin{array}{l}50 \\
444 \\
\end{array}$ & \begin{tabular}{|l|}
10.1 \\
89.9 \\
\end{tabular} & $\begin{array}{l}11.94 \pm 1.64 \\
11.75 \pm 0.48\end{array}$ & \begin{tabular}{|l|}
237.80 \\
248.59 \\
\end{tabular} & $Z=-0.507$ & 0.507 \\
\hline \multicolumn{8}{|l|}{ Preferred preventive measure } \\
\hline Mask & $\begin{array}{l}\text { Yes } \\
\text { No }\end{array}$ & $\begin{array}{l}474 \\
20 \\
\end{array}$ & \begin{tabular}{|l|}
96.0 \\
4.0 \\
\end{tabular} & $\begin{array}{l}11.93 \pm 0.47 \\
8.15 \pm 1.83 \\
\end{array}$ & \begin{tabular}{|l|}
249.80 \\
192.93 \\
\end{tabular} & $Z=-1,747$ & 0.081 \\
\hline Distance (1 meter) & $\begin{array}{l}\text { Yes } \\
\text { No }\end{array}$ & $\begin{array}{l}445 \\
49 \\
\end{array}$ & \begin{tabular}{|l|}
90.1 \\
9.9 \\
\end{tabular} & $\begin{array}{l}11.90 \pm 0.49 \\
10.63 \pm 1.40\end{array}$ & \begin{tabular}{|l|}
249.44 \\
229.88 \\
\end{tabular} & $Z=-0.912$ & 0.362 \\
\hline Disinfectant & $\begin{array}{l}\text { Yes } \\
\text { No }\end{array}$ & $\begin{array}{l}270 \\
224 \\
\end{array}$ & \begin{tabular}{|l|}
54.7 \\
45.3 \\
\end{tabular} & $\begin{array}{l}11.94 \pm 0.61 \\
11.57 \pm 0.71\end{array}$ & $\begin{array}{l}253.34 \\
240.46 \\
\end{array}$ & $Z=-1,000$ & 0.317 \\
\hline Glove & $\begin{array}{l}\text { Yes } \\
\text { No }\end{array}$ & $\begin{array}{l}259 \\
235 \\
\end{array}$ & $\begin{array}{l}52.4 \\
47.6 \\
\end{array}$ & $\begin{array}{l}11.97 \pm 0.65 \\
11.56 \pm 0.67 \\
\end{array}$ & \begin{tabular}{|l|}
250.16 \\
244.57 \\
\end{tabular} & $Z=-0.435$ & 0.663 \\
\hline Cologne & $\begin{array}{l}\text { Yes } \\
\text { No }\end{array}$ & $\begin{array}{l}229 \\
265 \\
\end{array}$ & \begin{tabular}{|l|}
46.4 \\
53.6 \\
\end{tabular} & $\begin{array}{l}12.84 \pm 0.72 \\
10.85 \pm 0.59\end{array}$ & $\begin{array}{l}260.99 \\
235.84 \\
\end{array}$ & $Z=-1,955$ & 0.051 \\
\hline Other (soap, visor, napkin, cleaning water) & $\begin{array}{l}\text { Yes } \\
\text { No }\end{array}$ & $\begin{array}{l}15 \\
479 \\
\end{array}$ & \begin{tabular}{|l|}
3.0 \\
97.0 \\
\end{tabular} & $\begin{array}{l}12.40 \pm 2.36 \\
11.75 \pm 0.47\end{array}$ & $\begin{array}{l}266.47 \\
246.91 \\
\end{array}$ & $Z=-0.523$ & 0.601 \\
\hline \multicolumn{8}{|l|}{ The most common emotion about COVID-19 } \\
\hline Concern & $\begin{array}{l}\text { Yes } \\
\text { No }\end{array}$ & $\begin{array}{l}413 \\
81 \\
\end{array}$ & \begin{tabular}{|l|}
83.6 \\
16.4 \\
\end{tabular} & $\begin{array}{l}12.04 \pm 0.51 \\
10.39 \pm 1.04\end{array}$ & $\begin{array}{l}251.09 \\
229.19 \\
\end{array}$ & $Z=-1,264$ & 0.206 \\
\hline Fear & $\begin{array}{l}\text { Yes } \\
\text { No }\end{array}$ & $\begin{array}{l}190 \\
304 \\
\end{array}$ & $\begin{array}{l}38.5 \\
61.5 \\
\end{array}$ & $\begin{array}{l}16.10 \pm 0.84 \\
9.07 \pm 0.48 \\
\end{array}$ & \begin{tabular}{|l}
305.05 \\
211.53 \\
\end{tabular} & $Z=-7,092$ & $<0.001$ \\
\hline Sadness & $\begin{array}{l}\text { Yes } \\
\text { No }\end{array}$ & $\begin{array}{l}167 \\
327 \\
\end{array}$ & \begin{tabular}{|l|}
33.8 \\
66.2 \\
\end{tabular} & $\begin{array}{l}14.21 \pm 0.85 \\
10.53 \pm 0.53\end{array}$ & $\begin{array}{l}281.94 \\
229.91 \\
\end{array}$ & $Z=-3,837$ & $<0.001$ \\
\hline Difficulty in coping & $\begin{array}{l}\text { Yes } \\
\text { No }\end{array}$ & $\begin{array}{l}107 \\
387 \\
\end{array}$ & $\begin{array}{l}21.7 \\
78.3 \\
\end{array}$ & $\begin{array}{l}19.08 \pm 1.12 \\
9.75 \pm 0.45\end{array}$ & $\begin{array}{l}343.80 \\
220.87 \\
\end{array}$ & $Z=-7,893$ & $<0.001$ \\
\hline $\begin{array}{l}\text { Other (communication difficulties, } \\
\text { confusion, uncertainty, anger) }\end{array}$ & $\begin{array}{l}\text { Yes } \\
\text { No }\end{array}$ & $\begin{array}{l}38 \\
456 \\
\end{array}$ & $\begin{array}{l}7.7 \\
92.3 \\
\end{array}$ & $\begin{array}{l}12.92 \pm 1.70 \\
11.68 \pm 0.48\end{array}$ & $\begin{array}{l}262.38 \\
246.26 \\
\end{array}$ & $Z=-0.670$ & 0.503 \\
\hline \multicolumn{8}{|l|}{ Coping methods in social isolation } \\
\hline Kitchen activities (food, cake, etc.) & $\begin{array}{l}\text { Yes } \\
\text { No }\end{array}$ & $\begin{array}{l}341 \\
153\end{array}$ & $\begin{array}{l}69.0 \\
31.0\end{array}$ & $\begin{array}{l}11.93 \pm 0.54 \\
11.42 \pm 0.90\end{array}$ & $\begin{array}{l}253.00 \\
235.24\end{array}$ & $Z=-1.281$ & 0.200 \\
\hline
\end{tabular}




\begin{tabular}{|c|c|c|c|c|c|c|c|}
\hline \multicolumn{2}{|l|}{ Variables } & & \multirow{2}{*}{$\begin{array}{c}\% \% \\
100\end{array}$} & \multirow{2}{*}{$\begin{array}{l}\text { BAI } \\
X \pm S D\end{array}$} & \multicolumn{3}{|l|}{ Significance } \\
\hline & & & & & Mean rank & Test & p-value \\
\hline Game and painting activities with children & $\begin{array}{l}\text { Yes } \\
\text { No }\end{array}$ & $\begin{array}{l}338 \\
106\end{array}$ & $\begin{array}{l}68.4 \\
31.6\end{array}$ & $\begin{array}{l}11.95 \pm 0.56 \\
11.38 \pm 0.84\end{array}$ & $\begin{array}{l}250.62 \\
240.73\end{array}$ & $Z=-0.717$ & 0.473 \\
\hline Home cleaning & $\begin{array}{l}\text { Yes } \\
\text { No }\end{array}$ & $\begin{array}{l}325 \\
169\end{array}$ & $\begin{array}{l}65.8 \\
34.2\end{array}$ & $\begin{array}{l}12.20 \pm 057 \\
10.94 \pm 0.78\end{array}$ & $\begin{array}{l}254.54 \\
233.97\end{array}$ & $Z=-1,521$ & 0.128 \\
\hline Reading & $\begin{array}{l}\text { Yes } \\
\text { No }\end{array}$ & $\begin{array}{l}200 \\
294\end{array}$ & $\begin{array}{l}40.5 \\
59.5\end{array}$ & $\begin{array}{l}10.67 \pm 0.70 \\
12.52 \pm 0.61\end{array}$ & $\begin{array}{l}230.99 \\
258.73\end{array}$ & $z=-2,123$ & 0.034 \\
\hline Professional activities (working from home) & $\begin{array}{l}\text { Yes } \\
\text { No }\end{array}$ & $\begin{array}{l}159 \\
335\end{array}$ & $\begin{array}{l}32.2 \\
67.8\end{array}$ & $\begin{array}{l}11.13 \pm 0.76 \\
12.08 \pm 0.58\end{array}$ & $\begin{array}{l}241.64 \\
250.28\end{array}$ & $Z=-0.629$ & 0.529 \\
\hline $\begin{array}{l}\text { Individual hobbies (guitar, stone painting, mandala, sports } \\
\text { events) }\end{array}$ & $\begin{array}{l}\text { Yes } \\
\text { No }\end{array}$ & $\begin{array}{l}116 \\
378\end{array}$ & $\begin{array}{l}23.5 \\
76.5\end{array}$ & $\begin{array}{l}11.59 \pm 0.89 \\
11.83 \pm 0.54\end{array}$ & $\begin{array}{l}248.64 \\
247.15\end{array}$ & $Z=-0.099$ & 0.921 \\
\hline
\end{tabular}

Table IV. The effect of some parents-related variables on their anxiety scores $(n=494)$

Dependent variable: the anxiety level

\section{Independent variables: working status, chronic disease, caregiver, most common emotions}

Constant

Working status

(Working Not working)

Presence of chronic disease in family members

(Yes No)

Caregiver for children in the COVID-19 pandemic

(Mother/Father Caregiver Grandma/grandfather Other)

The most common emotion about COVID-19

Concern (Yes No)

The most common emotion about COVID-19

Fear (Yes No)

The most common emotion about COVID-19

Sadness (Yes No)

The most common emotion about COVID-19

Difficulty in coping (Yes No)

$\mathrm{R}$

$R^{2}$

F

$p^{*}$

${ }^{*} p<0.05$

B: Fixed value/regression load (Sabit değer/regresyon yükü), SE: Standard error, Beta: Standardized regression load (Standartlaştırılmış regresyon yükü), COVID-19: Coronavirus Disease-2019

COVID-19 pandemic, these symptoms levels increased after quarantine (27). Restrictive prevention and isolation can be confusing, and not well understood for children (15). It is considered common for children and their families to experience anxiety, panic, and fear, both for themselves and their families, friends, loved ones, and relatives (28). In this study, the most common emotions experienced by parents in the process of COVID-19 were concern/anxiety (83.6\%), fear $(38.5 \%)$, sadness $(33.8 \%)$, and difficulty in coping (21.7\%). In studies on this subject, it has been stated that the features seen in individuals in the process of COVID19 are often paranoia, sadness, fear, anxiety, anger, and depression $(13,29,30)$. In this study, $90.3 \%$ of parents stayed at home (social isolation or quarantine). In a study on global 
epidemics, it was stated that people who were isolated for 2 weeks because they were in contact with MERS showed anxiety signs and anger such as fear, isolation, and social withdrawal (31). In addition to isolation or quarantine, anxiety levels were found to be high in both parents and their children during the pandemic in cases of chronic illness and long-term regular medication.

If parents experience situations such as anxiety, fear, sadness, and difficulty in coping, it can negatively affect their children's health. In this situation, families are advised to talk to their children about how they keep them safe and to try to manage any stress they feel (19). In this study, the coping mechanism of the parents during their stay at home to manage their anxiety for themselves included kitchen activities (cooking, baking cake, etc.) (69.0\%), games and painting activities with their children (68.4\%), home cleaning $(65.8 \%)$, and reading $(40.5 \%)$. The anxiety score average of those parents who preferred reading was lower. In another study, similarly, it was stated that children prefer activities such as physical entertainment or reading during the epidemic process (28). Additionally, it is recommended that parents teach their children handwashing techniques or how to apply hand disinfectants and regularly wash any contacted surfaces with house cleaning materials so that the children and parents can cope with this process (19). In this study, it was found that parents preferred masks, social-distancing (1 meter), disinfectants, gloves, and cologne as first-line methods of protection. In another study, it was also found that parents attach importance to physical distancing especially in interpersonal relationships (29). Since diseases that are transmitted by the respiratory way require a specific intimacy between people, social-distancing reduces transmission (32). UNICEF has prepared a guide especially for those parents with small children during the COVID-19 period. This guide contains topics such as the importance of handwashing, the importance of not touching faces with dirty hands, the use of masks, keeping one meter social distance, and avoiding crowded environments (33).

\section{Study Limitations}

This study was cross-sectional and it had some limitations. The study particularly focused on those parents who used social media and the sample was limited only to those who used social media; therefore, homogeneity of the participants could not be achieved. Some parents may have limited internet access or not have had time due to their children.

\section{Conclusion}

In this study, it was found that the most common emotion experienced by the parents was fear, anxiety, sadness, and difficulty in coping and mild anxiety levels. It is important to research the psychological effects of international epidemics on populations and to create strategies for reducing negative psychological effects. It is recommended to develop programs to support parents during quarantine to prevent and alleviate the psychological effects of the pandemic by starting social and medical initiatives. As the COVID-19 pandemic affects the whole of society, it also affects children and their parents who are one of the risk groups by creating fear and anxiety. It is thought that planning studies, intervention programs, and training that will strengthen the mental health of their children can be effective in managing any negative effects. Nurses working in the field of paediatric nursing, family health nursing or public health nursing should have a responsibility in this part. It is recommended to conduct studies that can identify the causes of anxiety and stress in order to be prepared for situations such as epidemics that negatively affect children, families and society. In addition, studies which determine the problems faced by people during the quarantine process will be effective in examining this issue in depth.

\section{Ethics}

Ethics Committee Approval: İzmir Bakırçay University, Non-Interventional Clinical Research Ethics Committee's written permission was taken (approval number: 21/21, approval date: 20.04.2020) to conduct the research.

Informed Consent: The consents of the individuals were taken and then they were allowed to answer the questions after the consent.

Peer-review: Externally peer-reviewed.

\section{Authorship Contributions}

Concept: Ş.B.Y., A.K., M.G., Design: Ş.B.Y., A.K., M.G., Data Collection or Processing: S..B.Y., A.K., M.G., Analysis or Interpretation: Ş.B.Y., Writing: Ş.B.Y., A.K., M.G. Critical Revision: Ş.B.Y., A.K., M.G.

Conflict of Interest: The authors declared no conflict of interest.

Financial Disclosure: The authors declared that this study received no financial support.

\section{References}

1. Adhikari SP, Meng S, Wu YJ, et al. Epidemiology, causes, clinical manifestation and diagnosis, prevention and control 
of coronavirus disease (COVID-19) during the early outbreak period: A scoping review. Infect Dis Poverty 2020; 9:29.

2. Li Y, Guo F, Cao Y, Li L, Guo Y. Insight into COVID-2019 for pediatricians. Pediatric Pulmonology 2020; 55:1-4.

3. WHO. Technical guidance, 2020a. 18 March 2020 archived at https://www.who.int/emergencies/diseases/novelcoronavirus-2019/technical-guidance/naming-thecoronavirus-disease-(covid-2019)-and-the-virus-that-causesit).

4. Mahase E. China coronavirus: WHO declares international emergency as death toll exceeds 200. BMJ 2020; 368:408.

5. Horton R. Offline: 2019-nCoV- "A desperate plea". Lancet 2020; 395:400.

6. Ministry of Health. Life Fits Home, 2020. (30 April 2020 archived at https://covid19bilgi.saglik.gov.tr/tr/videolar.html)

7. Ko CH, Yen CF, Yen JY, Yang MJ. Psychosocial impact among the public of the severe acute respiratory syndrome epidemic in Taiwan. Psychiatry Clin Neurosci 2006; 60:397-403.

8. Peng EY, Lee MB, Tsai ST, et al. Population-based post-crisis psychological distress: an example from the SARS outbreak in Taiwan. J Formos Med Assoc 2010; 109:524-32.

9. Taha SA, Matheson K, Anisman H. H1N1 was not all that scary: Uncertainty and stressor appraisals predict anxiety related to a coming viral threat. Stress Health 2014; 30:149-57.

10. Hawryluck L, Gold WL, Robinson S, Pogorski S, Galea S, Styra R. SARS Control and Psychological Effects of Quarantine, Toronto, Canada. Emerg Infect Dis 2004; 10:1206-12.

11. Shultz JM, Baingana F, Neria Y. The 2014 Ebola outbreak and mental health: current status and recommended response. JAMA 2015; 313:567-8.

12. Wang C, Pan R, Wan X, et al. Immediate psychological responses and associated factors during the initial stage of the 2019 coronavirus disease (COVID-19) epidemic among the general population in China. Int I Environ Res Public Health 2020; 17:1729.

13. Brooks SK, Webster RK, Smith LE, et al. The psychological impact of quarantine and how to reduce it: Rapid review of the evidence. Lancet 2020; 395:912-20.

14. Coyne LW, Gould ER, Grimaldi M, Wilson KG, Baffuto G, Biglan A. First Things First: Parent Psychological Flexibility and SelfCompassion During COVID-19. Behav Anal Pract 2020; 14:10928.

15. Vessey JA, Betz CL. Everything old is new again: COVID-19 and public health. J Pediatr Nurs 2020; 52:7-8.

16. Fraser S, Grant J, Mannix T. The role and experience of child and family health nurses in developed countries: a review of the literature. Neonatal, Paediatric and Child Health Nursing 2014; 17:2-10.

17. Coulson N. Online Research Methods for Psychologists. Red Globe Press, 2015.

18. Qualtrics. Determining Sample Size: How to Ensure You Get the Correct Sample Size. (21 April 2020, archived at https://www. qualtrics.com/experience-management/research/determinesample-size/)
19. Thompson LA, Rasmussen SA. What does the Coronavirus Disease 2019 (COVID-19) mean for families? JAMA Pediatr 2020; 174:628.

20. WHO. Technical guidance, 2020b. (18 March 2020, archived at https://www.who.int/emergencies/diseases/ novel-coronavirus-2019/technical-guidance/naming-thecoronavirus-disease-(covid-2019)-and-the-virus-that-causes-it Accessed)

21. WHO. Coronavirus disease Situation Report 58, 2020c. (18 March 2020, archived at https://www.who.int/docs/defaultsource/coronaviruse/situation-reports/20200318-sitrep-58covid-19.pdf?sfvrsn=20876712_2)

22. Ulusoy M. Sahin NH, Erkmen H. Turkish version of the Beck Anxiety Inventory: Psychometric Properties. Journal of Cognitive Psychotherapy 1998; 12:163-72.

23. Shigemura I, Ursano RJ, Morganstein JC, Kurosawa M, Benedek DM. Public responses to the novel 2019 coronavirus (2019-nCoV) in Japan: Mental health consequences and target populations. Psychiatry Clin Neurosci 2020; 74:281-2.

24. Cava MA, Fay KE, Beanlands HJ, McCay EA, Wignall R. The experience of quarantine for individuals affected by SARS in Toronto. Public Health Nurs 2005; 22:398-406.

25. Röhr S, Müller F, Jung F, Apfelbacher C, Seidler A, Riedel-Heller SC. Psychosocial impact of quarantine measures during serious coronavirus outbreaks: A rapid review. Psychiatr Prax 2020; 47:179-89.

26. Asmundson GJG, Taylor S. Chronophobia: Fear and the 2019nCoV outbreak. ) Anxiety Disord 2020; 70:102-96.

27. Ozamiz-Etxebarria N, Dosil-Santamaria M, PicazaGorrochategui M, Idoiaga-Mondragon N. Stress, anxiety, and depression levels in the initial stage of the COVID-19 outbreak in a population sample in the northern Spain. Cad Saude Publica 2020; 36:e00054020

28. Jiao WY, Wang LN, Liu J, et al. Behavioral and Emotional Disorders in Children during the COVID-19. Epidemic. Journal Pediatr 2020; 221:264-6.

29. Kundu B, Bhowmik D. Societal impact of novel coronavirus (COVID-19 pandemic) in India. 2020. DOI: 10.31235/osf.io/vm5rz

30. Nicomedes CJC, Avila RMA. An Analysis on the Panic of Filipinos during COVID-19 Pandemic in the Philippines. Journal of Economic Studies 2020. DOI: 10.13140/RG.2.2.17355.54565.

31. Jeong H, Yim HW, Song YJ, et al. Mental health status of people isolated due to Middle East Respiratory Syndrome. Epidemiol Health 2016; 38:e2016048.

32. Wilder-Smith A, Freedman DO. Isolation, quarantine, social distancing and community containment: pivotal role for oldstyle public health measures in the novel coronavirus (2019nCoV) outbreak. J Travel Med 2020; 27:taa020. doi: 10.1093/jtm/ taaa020

33. UNICEF. Coronavirus Explained to Parents of Young Children, 2020. (30 April 2020, archived at https://www.unicef.org/ southafrica/media/2801/file/ZAF-COVID-19-explained-toparents-of-young-children-2020-ENG.pdf) 